\title{
Влияние эффекта электростатического экранирования на фотоэлектрические свойства гетероструктур с глубокими квантовыми ямами
}

\author{
(С) Л.В. Данилов, М.П. Михайлова, И.А. Андреев, Г.Г. Зегря \\ Физико-технический институт им. А.Ф. Иофрфе Российской академии наук, \\ 194021 Санкт-Петербург, Россия \\ E-mail: danleon84@mail.ru
}

(Получена 6 фревраля 2017 г. Принята к печати 14 февраля 2017 г.)

Работа посвящена теоретическому исследованию влияния электростатического потенциала, индуцированного носителями заряда одного знака, локализованными в глубокой квантовой яме, на вольт-амперные характеристики фотоприемных гетероструктур. На примере $p-i-n$-структуры с одиночной глубокой квантовой ямой для электронов, находящейся в $i$-области, показано, что экранирование внешнего приложенного электрического поля приводит к увеличению дифференциальной фотопроводимости такой гетероструктуры по сравнению с $p-i-n$-структурой без промежуточного двумерного слоя.

DOI: 10.21883/FTP.2017.09.44883.8509

\section{1. Введение}

Исследуемый эффект является общим для гетероструктур с глубокими квантовыми ямами на основе таких соединений, как $\mathrm{GaSb} / \mathrm{InAs}(\mathrm{Sb}), \mathrm{GaSb} / \mathrm{AlSb}$, $\operatorname{InAs}(\mathrm{Sb}) / \mathrm{Al}(\mathrm{As}) \mathrm{Sb}$, и связан с изменением зонной диаграммы гетероструктуры за счет экранирования внешнего электрического поля электронами, локализованными в квантовой яме.

Следует отметить, что подобная локализация заряда одного знака в гетероструктурах с глубокими квантовыми ямами может происходить не только при фотовозбуждении, но и при инжекции носителей заряда в активную область гетероструктуры под действием внешнего (прямого) электрического поля. Такая неравномерная инжекция носителей заряда приводит к преобладанию внутрибарьерных излучательных переходов, что негативно сказывается на рабочих характеристиках светоизлучающих структур, снижая их квантовую эффективность [1]. Основной причиной неравномерной инжекции в гетероструктурах с высокими потенциальными барьерами является туннельный механизм переноса носителей заряда, что приводит к фильтрации эффективной массы, т.е. перенос заряда осуществляется преимущественно электронами с малой по сравнению с дырками эффективной массой. Впервые на это обстоятельство было обращено внимание в работе [2] по исследованию фотопроводимости в сверхрешетках AlInAs/GaInAs.

Другой важной особенностью гетероструктур с высокими потенциальными барьерами и глубокими квантовыми ямами, оказывающей непосредственное влияние на их люминесцентные и фотоэлектрические свойства, является высокая чувствительность характерного времени захвата носителей заряда к ширине квантовой ямы. В работе [3] рассмотрены различные механизмы захвата электронов в глубокую квантовую яму $\mathrm{AlSb} / \mathrm{InAsSb} / \mathrm{AlSb}$, и было показано, что в зависимости от положения уровней энергии в квантовой яме время захвата электронов может варьироваться от $10^{-13}$ до $10^{-11}$ с. При этом в некоторых случаях кулоновский захват электронов может преобладать над захватом при рассеянии на оптических фононах. Уменьшение характерного времени захвата, с одной стороны, способствует увеличению эффективности заполнения активной области гетероструктуры, что является положительным фактором для светоизлучающих структур, однако, с другой стороны, способствует увеличению экранирующего поля, направленного навстречу внешнему приложенному смещению, что может негативно отразиться на фотоэлектрических характеристиках гетероструктуры. Особенно активно эффект электростатического экранирования должен проявляться в гетероструктурах II типа, где носители заряда разных знаков разделены гетерограницей. При этом создание экранирующего потенциала может происходить как при пространственной локализации электронов, так и при локализации дырок. В работе [4] впервые наблюдался сверхлинейный рост фототока при увеличении обратного смещения в объемных гетероструктурах на основе изотипного гетероперехода II типа $n$-GaSb/n-GaInAsSb. Обнаруженный эффект был объяснен локализацией генерируемых при засветке дырок в самосогласованной квантовой яме на гетерогранице. При этом дополнительный положительный заряд, создаваемый дырками в области объемного заряда $n$-GaSb, способствовал уменьшению толщины диффузионного барьера на гетерогранице, что в свою очередь привело к увеличению туннельного тока электронов сквозь него.

\section{2. Основные уравнения}

Для исследования эффекта электростатического экранирования в гетероструктурах с двумерными слоями рассмотрим анизотипную гетероструктуру II типа $n$-GaSb/InAs/p-GaSb с одиночной квантовой ямой InAs 
для электронов (рис. 1). Внешнее электрическое поле приложено в обратном внутреннему полю направлении („,+“ к n-слою, „-“" к p-слою). Засветка осуществляется со стороны $p$-слоя. При этом будем считать, что $p$-слой достаточно толстый, так что весь световой поток полностью поглощается в нем.

При увеличении обратного напряжения время пролета слоя InAs электронами, генерируемыми при засветке в слое $p$-GaSb, уменьшается, что приводит к уменьшению коэффициента захвата электронов в квантовую яму. В то же время общий ток электронов через двумерный слой увеличивается, что способствует наращиванию концентрации электронов в квантовой яме и, следовательно, способствует увеличению экранирующего электростатического потенциала слоя InAs. Для того чтобы оценить вклад каждого из этих факторов, необходимо найти зависимость общего фототока в структуре от напряженности электрического поля $E(x)$. Для определенности будем считать, что при обратном смещении основной вклад в общий фототок вносит дрейфовая составляющая $j(x)=e \mu n(x) E(x)$. Направление $x$ удобно отсчитывать справа налево по направлению тока электронов от правой границы квантовой ямы. В этом случае уравнение непрерывности для фототока через слой InAs выглядит следующим образом:

$$
\mu\left[n(x) \frac{d E}{d x}+E(x) \frac{d n}{d x}\right]=-\frac{n(x)}{\tau},
$$

где $n(x)$ - концентрация электронов, инжектируемых из слоя $p$-GaSb, $\tau$ - характерное время захвата электронов сплошного спектра в квантовую яму, $\mu-$ подвижность электронов в $\mathrm{GaSb}$. Интегрируя уравнение (1), получим зависимость плотности электронов сплошного спектра слоя InAs от напряженности электрического поля:

$$
n(x)=n_{0} \frac{E(0)}{E(x)} \exp \left(-\int_{0}^{x} \frac{d x}{\mu \tau E(x)}\right),
$$

где $n_{0}=G \tau_{r}$ - концентрация электронов на гетрогранице InAs/ $p$-GaSb, определяемая интенсивностью засветки $G$ и временем жизни носителей заряда $\tau_{r}$ в слое $p-\mathrm{GaSb}$.

Домножив уравнение (2) на $е \mu E(x)$, получаем выражение для плотности фототока через слой InAs:

$$
j(x)=j_{0} \exp \left(-\int_{0}^{x} \frac{d x}{\mu \tau E(x)}\right),
$$

$j_{0}$ представляет собой классическую линейную зависимость плотности фототока от напряженности электрического поля в фотодиодах на объемных $p-n$-структурах, $j_{0}=e \mu n_{0} E(0)$. Коэффициент $\exp \left[-\int_{0}^{d_{0}} d x / \mu \tau E(x)\right]$ определяет долю электронов, прошедших слой InAs без захвата в квантовую яму, где $d_{0}-$ ширина квантовой ямы. Соответственно коэффициент $1-\exp \left[-\int_{0}^{d_{0}} d x / \mu \tau E(x)\right]$

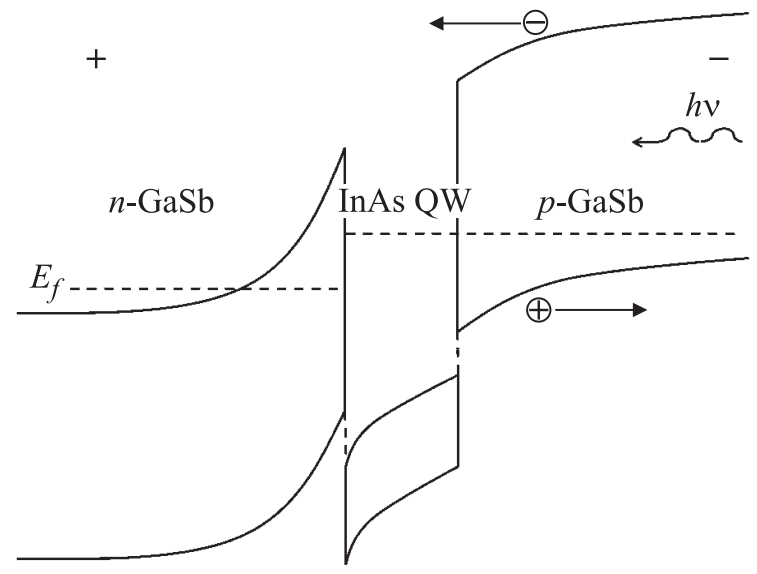

Рис. 1. Зонная диаграмма гетероструктуры $n$-GaSb/InAs/ $p$-GaSb с одиночной глубокой квантовой ямой $(\mathrm{QW})$ для электронов при обратном смещении. $E_{f}-$ уровень Ферми.

характеризует долю захваченных в квантовую яму электронов. Полный ток через структуру $j_{0}$ является суммой дрейфового тока через двумерный слой, определяемого выражением (3), и туннельного тока через диффузионный барьер на гетерогранице $n$-GaSb/InAs. Как отмечалось ранее, туннельный ток через гетерограницу является важной составляющей общего инжекционного тока в структурах с высокими потенциальными барьерами и играет ключевую роль в процессах электролюминесценции в светоизлучающих структурах [5]. В нашем же случае туннельный ток через интерфейс $n$-GaSb/InAs наряду с коэффициентом захвата определяет концентрацию электронов, локализованных в квантовой яме. В упрощенном виде выражение для туннельного тока можно записать следующем образом:

$$
j_{\text {tun }}=e T\left(k_{x}\right) v_{x} \frac{n^{2 \mathrm{D}}}{d_{0}},
$$

где $T\left(k_{x}\right)$ - коэффициент туннельной прозрачности, зависящий от квазиимпульса электронов в направлении $x$, перпендикулярном плоскости гетерограницы, величина $v_{x}$ соответствует $x$-компоненте скорости электронов, локализованных в квантовой яме вблизи гетерограницы $n$-GaSb/InAs, $m_{c}$ - эффективная масса электрона в $\mathrm{InAs}, n^{2 \mathrm{D}}$ - двумерная концентрация электронов в квантовой яме.

Концентрацию электронов, локализованных в квантовой яме, можно найти, сопоставив выражение (4) с потоком электронов, захваченных в квантовую яму,

$$
j_{0}\left\{1-\exp \left[-\int_{0}^{d_{0}} \frac{d x}{\mu \tau E(x)}\right]\right\}=e T\left(k_{x}\right) v_{x} \frac{n^{2 \mathrm{D}}}{d_{0}} .
$$

В общем случае напряженность электрического поля $E(x)$ зависит от вида волновой функции электронов в 
квантовой яме $\Psi(x)$, которая в свою очередь определяется профилем потенциальной энергии $-e \varphi(x)$. Таким образом, для определения зависимости $E(x)=-d \varphi / d x$ необходимо найти решение самосогласованной системы уравнений Шредингера-Пуассона $[6,7]$

$$
\left\{\begin{array}{l}
-\frac{\hbar^{2}}{2 m_{c}} \nabla^{2} \Psi(x)-e \varphi(x) \Psi(x)=E_{n} \Psi(x), \\
\frac{d^{2} \varphi}{d x^{2}}=\frac{e}{\varepsilon} n^{2 \mathrm{D}}|\Psi(x)|^{2}
\end{array}\right.
$$

с граничными условиями, налагаемыми на вектор электрической индукции,

$$
-\left.\varepsilon\left(d_{0}^{+}\right) \frac{d \varphi}{d x}\right|_{d_{0}^{+}}=-\left.\varepsilon\left(d_{0}^{-}\right) \frac{d \varphi}{d x}\right|_{d_{0}^{-}}
$$

и

$$
-\left.\varepsilon\left(0^{+}\right) \frac{d \varphi}{d x}\right|_{0^{+}}=-\left.\varepsilon\left(0^{-}\right) \frac{d \varphi}{d x}\right|_{0^{-}} .
$$

Стоит отметить, что в системе (6) под $n^{2 \mathrm{D}}$ подразумевается полная концентрация электронов, локализованных в квантовой яме, т.е. не только электронов, захваченных из фототока, но и электронов, захваченных при диффузии из $n$-GaSb. В результате для напряженности электрического поля в области квантовой ямы получаем выражение

$$
E(x)=-\frac{e}{\varepsilon} n^{2 \mathrm{D}} \int|\Psi(x)|^{2} d x,
$$

где $\varepsilon$ - диэлектрическая проницаемость InAs. Подставляя выражение (7) в уравнение (5), можно найти зависимость $n^{2 \mathrm{D}}(E(0))$, а также полный фототок $j_{0}=j(E(0))$.

\section{3. Модель расчета для $p-i-n$-структуры с квантовой ямой}

Реализация алгоритма расчета, предложенного в предыдущем параграфе, является достаточно сложной задачей для компьютерного моделирования. Однако для качественного объяснения исследуемого эффекта достаточно рассмотреть простую модель одиночной квантовой ямы в однородном электрическом поле (рис. 2). Такая модель описывает поведение фототока в $p-i-n$-структуре с достаточно широкой областью собственной проводимости, шириной $L_{i}$, содержащей квантовую яму шириной $d_{0}$. В случае, если $L_{i} \gg d_{0}$, напряженность электрического поля в области квантовой ямы можно считать не зависящей от координаты и записать в виде суммы $E=E_{\text {ext }}+E_{s}$, где первое слагаемое соответствует внешнему электрическому полю, приложенному к гетероструктуре, а второе слагаемое отвечает экранирующему полю, создаваемому электронами, захваченными в квантовую яму, $\left|E_{s}\right|=2 \pi e n^{2 \mathrm{D}} / \varepsilon$. Выражение для $E_{s}$ может быть получено из теоремы Гаусса-Остроградского, если квантовую яму представить как бесконечную равномерно заряженную плоскость с плотностью поверхностного заряда $e n^{2 \mathrm{D}}$.

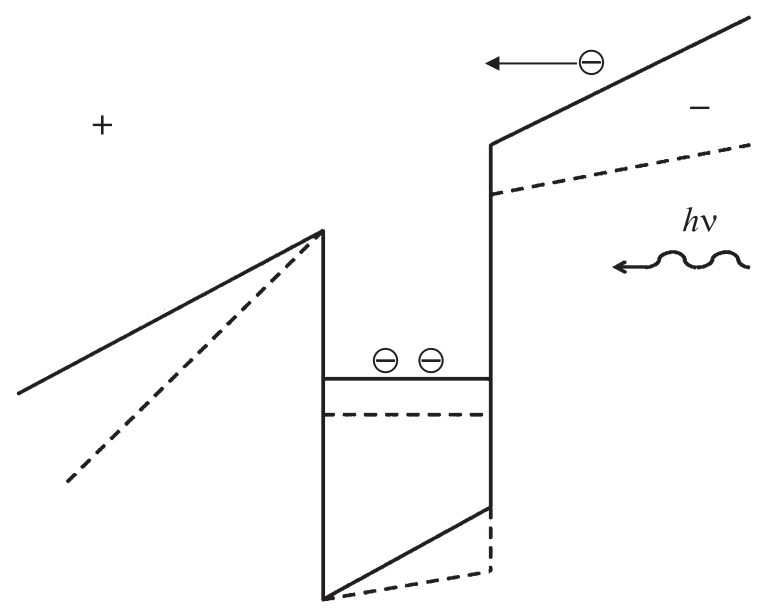

Рис. 2. Зонная диаграмма гетероструктуры с одиночной глубокой квантовой ямой для электронов в однородном электрическом поле. Штриховой линией показано изменение зонной диаграммы гетероструктуры под действием экранирующего электростатического потенциала электронов, локализованных в квантовой яме.

На рис. 2 штриховой линией показано изменение зонной диаграммы с учетом экранирующего потенциала квантовой ямы. Из представленной схемы видно, что, с одной стороны, накопление электронов в квантовой яме приводит к уменьшению напряженности электрического поля в широкозонной области справа от квантовой ямы, тем самым уменьшая плотность дрейфового тока фотоэлектронов, налетающих на яму, а с другой способствует увеличению напряженности электрического поля в области слева от квантовой ямы. Последнее обстоятельство приводит к уменьшению толщины потенциального барьера для электронов, туннелирующих из квантовой ямы. Зависимость коэффициента туннельной прозрачности треугольного барьера, образованного под действием однородного электрического поля $E$, может быть найдена в рамках квазиклассического приближения [8]:

$$
T(E) \approx \exp \left[-\frac{4 \sqrt{2 m_{c}}}{3 e \hbar E}\left(U-E_{n}\right)^{3 / 2}\right],
$$

где $U$ - высота потенциальной стенки квантовой ямы, а $E_{n}$ - энергетический уровень размерного квантования электронов (в дальнейшем будем иметь в виду основной уровень энергии). Штарковский сдвиг уровня $E_{n}$ в электрическом поле будем считать незначительным. Оценки, сделанные в рамках теории возмущений для небольших электрических полей, до $5 \cdot 10^{3} \mathrm{~B} / \mathrm{cm}$, дают поправки к энергии основного уровня, не превышающие 10 мэВ.

Теперь, принимая во внимание выражения (8), можно найти зависимость двумерной концентрации электронов в квантовой яме от напряженности внешнего электрического поля. Для этого в трансцендентном уравнении (5) заменим $E(x)$ на $E_{\text {ext }}+E_{\text {int }}-2 \pi e n^{2 \mathrm{D}} / \varepsilon$. 


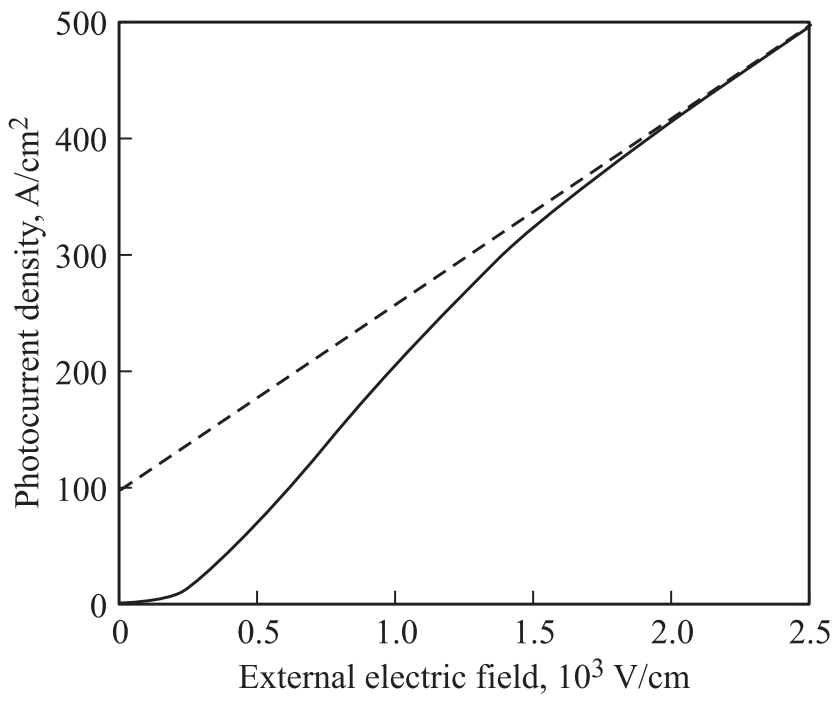

Pис. 3. Зависимость плотности фототока от напряженности внешнего электрического поля, приложенного к $p-i-n$-структуре на основе GaSb с одиночной глубокой квантовой ямой из InAs в $i$-области. Штриховой линией показана зависимость фототока от напряженности электрического поля в $p-i-n$-структуре без квантовой ямы.

В свою очередь, в выражении (8) напряженность экранирующего поля необходимо взять с обратным знаком, $E=E_{\mathrm{ext}}+E_{\mathrm{int}}+2 \pi e n^{2 \mathrm{D}} / \varepsilon$. Здесь для более точного соответствия рассматриваемой упрощенной модели и реальной ситуации мы ввели некоторое постоянное внутреннее поле $E_{\text {int }}$ по аналогии с полем, создаваемым областями объемного заряда в полупроводниках $n$ - и $p$-типа проводимости (рис. 1). При этом будем считать, что направление вектора напряженности внутреннего поля совпадает с направлением внешнего приложенного поля, как в случае обратносмещенной $p-i-n$-структуры, работающей в режиме фотодиода.

Зная зависимость $n^{2 \mathrm{D}}\left(E_{\text {ext }}\right)$, не составляет труда найти полный ток электронов в структуре:

$$
j_{0}=e n_{0} \mu\left[E_{\mathrm{ext}}+E_{\mathrm{int}}-\frac{2 \pi e n^{2 \mathrm{D}}\left(E_{\mathrm{ext}}\right)}{\varepsilon}\right] .
$$

На рис. 3 представлена зависимость полного фототока от напряженности внешнего электрического поля в $p-i-n$-структуре на основе $\mathrm{GaSb}$ с глубокой квантовой ямой из InAs, расположенной в $i$-области. В расчете использовались следующие значения параметров: $U=0.95$ эВ, $E_{n}=0.3$ эВ, $v_{x} \approx \sqrt{2 m_{c} E_{n} / \hbar^{2}}$ $=5 \cdot 10^{6} \mathrm{~cm} / \mathrm{c}, \quad \tau=10^{-12} \mathrm{c}, \quad \mu=10^{3} \mathrm{~cm}^{2} \cdot \mathrm{B}^{-1} \cdot \mathrm{c}^{-1}$, $n_{0}=10^{15} \mathrm{~cm}^{-3}, \quad \varepsilon=15, \quad d=5 \mathrm{HM}, \quad E_{\mathrm{int}}=600 \mathrm{~B} / \mathrm{cm}$. Внутреннее поле $E_{\text {int }}$ соответствует ширине области собственной проводимости $13-14$ мкм, $E_{\text {int }} \approx E_{g}^{\mathrm{GaSb}} / L_{i}$ $\left(E_{g}^{\mathrm{GaSb}}\right.$ - ширина запрещенной зоны $\left.\mathrm{GaAs}\right)$.

Штриховой линией на рис. 3 показана зависимость фототока от напряженности электрического поля в структуре без квантовой ямы. Такая линейная вольтамперная зависимость характерна для фотопроводимости в обычной объемной $p-i-n$-структуре. При этом отсечка на оси тока соответствует плотности протекающего в структуре тока под действием внутреннего поля. Вольт-амперная характеристика для фототока в структуре с квантовой ямой ведет себя принципиально иным образом. При малых внешних смещениях (до $200 \mathrm{~B} / \mathrm{cm}$ ) фотопроводимость практически отсутствует, плотность фототока не превышает значения $10 \mathrm{~A} / \mathrm{cm}^{2}$. Дальнейшее увеличение напряженности электрического поля (до $1500 \mathrm{~B} / \mathrm{cm}$ ) приводит к резкому возрастанию фототока в структуре, после чего происходит переход в линейную вольт-амперную зависимость для структуры без квантовой ямы.

\section{4. Обсуждение результатов}

Для того чтобы лучше понять причины такого нелинейного роста фототока при малых внешних смещениях, необходимо построить зависимость напряженности экранирующего поля электронов $E_{s}=-2 \pi e n^{2 \mathrm{D}}\left(E_{\mathrm{ext}}\right) / \varepsilon$, локализованных в квантовой яме, от напряженности внешнего приложенного поля (рис. 4). Для сравнения штриховой линией показан рост внешнего электрического поля в сочетании с постоянным внутренним полем структуры, $E_{\text {ext }}+E_{\text {int }}$.

Из приведенной зависимости видно, что напряженность экранирующего поля при малых внешних смещениях (до $200 \mathrm{~B} / \mathrm{cm}$ ) растет соразмерно внешнему приложенному полю. Можно сказать, что число захваченных в квантовую яму электронов полностью

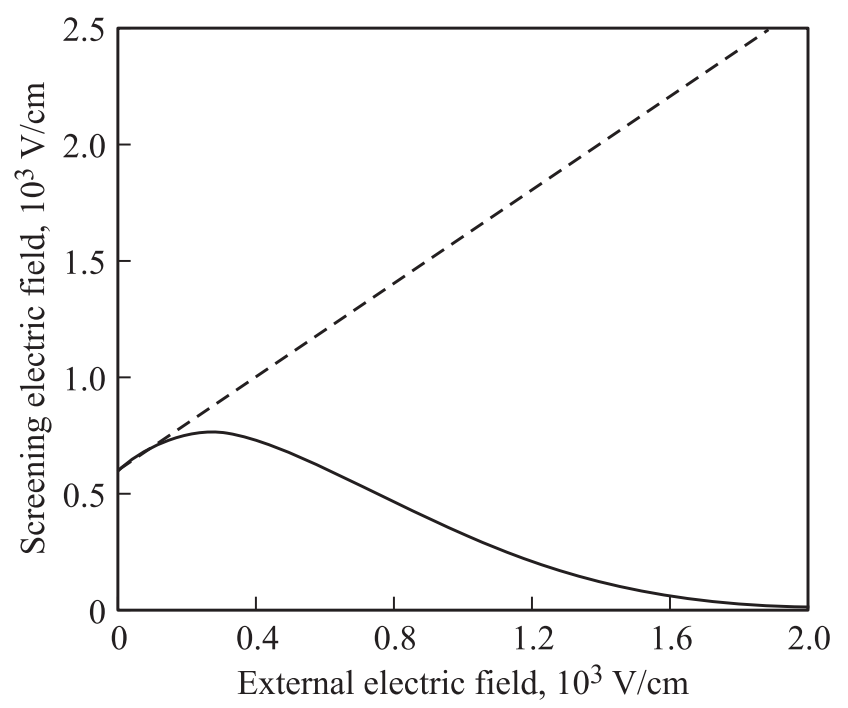

Рис. 4. Зависимость напряженности экранирующего поля от внешнего электрического поля, приложенного к $p-i-n$-структуре на основе $\mathrm{GaSb}$ с одиночной глубокой квантовой ямой из InAs в $i$-области. Штриховой линией показан рост внешнего электрического поля в сочетании с постоянным внутренним полем структуры. 


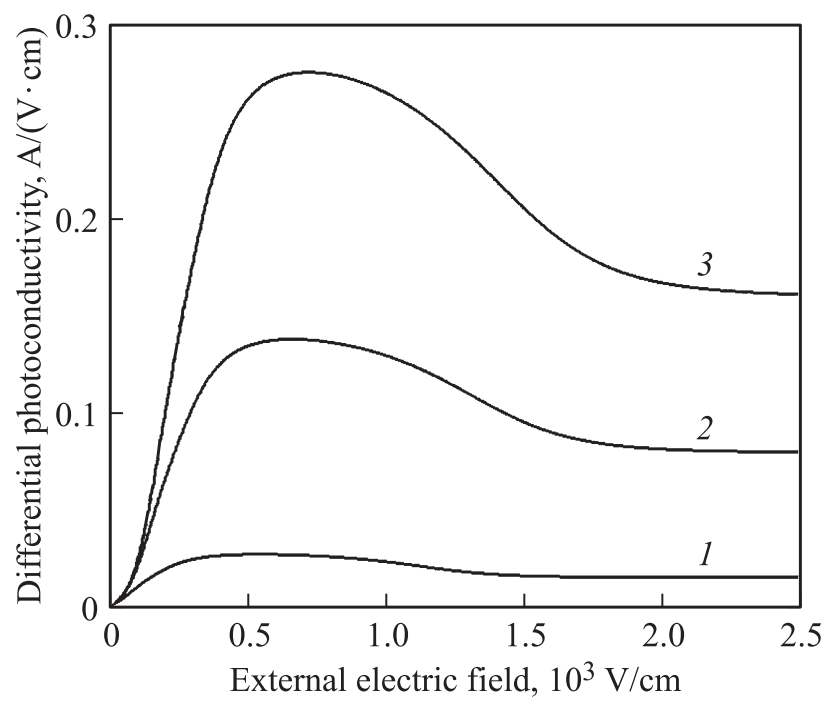

Рис. 5. Зависимость дифференциальной фотопроводимости от внешнего электрического поля, приложенного к $p-i-n$-структуре на основе $\mathrm{GaSb}$ с одиночной глубокой квантовой ямой из InAs в $i$-области. $n_{0}, \mathrm{~cm}^{-3}: 1-10^{14}$, $2-5 \cdot 10^{14}, 3-10^{15}$.

определяется током фотоэлектронов, проходящих через двумерный слой. При дальнейшем увеличении внешнего поля до $1500 \mathrm{~B} / \mathrm{cm}$ происходит быстрое уменьшение концентрации электронов в квантовой яме и, следовательно, уменьшение их экранирующего потенциала. Такое поведение объясняется суммарным действием двух процессов: уменьшением коэффициента захвата электронов в квантовую яму и увеличением туннельного тока электронов из квантовой ямы за счет уменьшения толщины потенциального барьера на левой гетерогранице (рис. 2). При этом общий ток электронов в системе резко возрастает. Быстрое уменьшение концентрации электронов в квантовой яме сменяется более плавным убыванием вплоть до нуля. При этом практически весь ток в системе будет определяться дрейфовым током электронов через двумерный слой так, как если бы квантовой ямы не было.

На рис. 5 представлена зависимость дифференциальной фотопроводимости рассматриваемой гетероструктуры, определяемой выражением $\gamma\left(E_{\text {ext }}\right)=d j_{0} / d E_{\text {ext}}$, от приложенного внешнего электрического поля. Видно, что максимальное значение фотопроводимости достигается при внешних смещениях 500-1000 В/см, при этом она линейно зависит от интенсивности засветки, которая определяет концентрацию электронов сплошного спектра вблизи правой границы квантовой ямы $n_{0}$. Участок кривых при внешних смещениях $>1500 \mathrm{~B} / \mathrm{cm}$ соответствует линейной резистивной фотопроводимости при отсутствии локализованных в двумерном слое электронов.

Таким образом, возвращаясь к анализу вольт-амперной характеристики (рис. 3), можно заключить, что гетероструктура с квантовой ямой, хотя и производит меньший по сравнению с объемной $p-i-n$-структурой фототок при малых внешних смещениях, что главным образом объясняется экранированием внутреннего электрического поля гетероструктуры, однако обладает большей удельной фотопроводимостью в определенном интервале внешних смешений, и это говорит о более высокой фоточувствительности таких гетероструктур.

\section{5. Заключение}

Описанный эффект увеличения фотопроводимости в гетероструктурах с глубокими квантовыми ямами может быть использован при создании высокочувствительных фотодетекторов с краем поглощения до 1.7 мкм, соответствующим ширине запрещенной зоны GaSb. При этом особый интерес представляют длины волн 1.55 и 1.3 мкм, отвечающие окнам прозрачности оптоволокна. Принимая во внимание, что высокий уровень чувствительности достигается при малых внешних смещениях, данный эффект можно рассматривать как основу для создания компактных фотоприемников для высокоскоростных коммуникационных систем с низким энергопотреблением. Также необходимо отметить, что предложенный в работе алгоритм расчета вольт-амперных характеристик может быть успешно обобщен на структуры с несколькими квантовыми ямами и структуры со сверхрешетками на основе соединений $\mathrm{InAs} / \mathrm{GaSb}$.

Работа частично поддержана грантами РФФИ № 16-08-01130 и 15-02-03151.

\section{Список литературы}

[1] L.V. Danilov, A.A. Petukhov, M.P. Mikhailova, E.V. Ivanov, K.V. Kalinina, G.G. Zegrya, N.D. Stoyanov, Yu.P. Yakovlev, A. Hospodkova, J. Pangrac, I. Oswald, M. Zikova, E. Hulicius. 23rd Int. Symp. „Nanostructures: Physics and Technology“ (St. Petersburg, 2015).

[2] F. Capasso, K. Mohammed, A.Y. Cho, R. Hull, A.L. Hutchinson. Phys. Rev. Lett., 50, 1152 (1985).

[3] Л.В. Данилов, Г.Г. Зегря. ФТП, 47 (10), 1347 (2013).

[4] И.А. Андреев, А.Н. Баранов, М.А. Мирсагатов, М.П. Михайлова, А.А. Рогачев, Г.М. Филаретова, Ю.П. Яковлев. Письма ЖТФ, 14 (5), 389 (1988).

[5] Л.В. Данилов, А.А. Петухов, М.П. Михайлова, Г.Г. Зегря, Э.В. Иванов, Ю.П. Яковлев. ФТП, 50 (6), 794 (2016).

[6] I.H. Tan, G.L. Snider, L.D. Chang, E.L. Hu. J. Appl. Phys., 68, 4071 (1990).

[7] P.N. Brounkov, T. Benyattou, G. Guillot. J. Appl. Phys., 80, 864 (1996).

[8] Л.Д. Ландау, Е.М. Лифшиц. Квантовая механика. Нерелятивистская теория (М., Физматлит, 1974).

Редактор Л.В. Шаронова 
Effect of electrostatic screening

on the photoelectric properties

of heterostructures with deep

quantum wells

L.V. Danilov, M.P. Mikhailova,

I.A. Andreev, G.G. Zegrya

loffe Institute,

194021 St. Petersburg, Russia

Abstract This work is devoted to theoretical study of electrostatic screening effect induced by charge carriers only one sign localized in deep quantum well. The influence of carrier screening on current-voltage characteristics in photoexcitation heterostructure was demonstrated. Using the simple model of $p-i-n$ structure with single deep quantum well placed in uniform electric field, it has been shown that the carrier screening effect helps to increase the differential photoconductivity in compare with bulk $p-i-n$ structure without intermediate 2D layer. 